\title{
Correlação entre o índice de massa corporal e os indicadores antropométricos de distribuição de gordura corporal em adultos e idosos
}

\section{Correlation between body mass index and body fat distribution anthropometric indices in adults and the elderly}

Lilian Ramos SAMPAIO ${ }^{1}$

Vanessa de Carvalho FIGUEIREDO²

\section{RE S U M O}

\section{Objetivo}

O objetivo deste trabalho foi avaliar a correlação entre o índice de massa corporal e indicadores antropométricos de distribuição de gordura em adultos e idosos.

\section{Métodos}

Trata-se de um estudo transversal, constituído por 634 indivíduos (316 adultos e 318 idosos) de ambos os sexos, atendidos nos ambulatórios do Hospital das Clínicas da Universidade Federal da Bahia (Salvador, BA). Mediram-se em cada indivíduo: peso, altura, pregas cutâneas triciptal e subescapular, circunferências de quadril e cintura, segundo técnicas propostas por Lohman, em 1988. Utilizou-se o teste de correlação de Pearson para avaliar a correlação entre o índice de massa corporal e os indicadores antropométricos de distribuição de gordura.

\section{Resultados}

As correlações entre o índice de massa corporal e a circunferência da cintura nos dois grupos etários do sexo masculino foram: de adultos $(r=0,93 ; p<0,001)$; de idosos $(r=0,89 ; p<0,001)$. Para os grupos do sexo feminino, as correlações foram: grupo de adultas $(r=0,93 ; p<0,001)$, de idosas $(r=0,86 ; p<0,001)$. A correlação entre o índice de massa corporal e a razão cintura quadril foi menor, mas estatisticamente significante, entre os dois grupos de cada sexo. Assim, para o sexo masculino, entre o grupo adulto $(r=0,64 ; p<0,001)$ e

\footnotetext{
1 Escola de Nutrição, Departamento de Ciências da Nutrição, Universidade Federal da Bahia. Av. Araújo Pinho, 31, Canela, 40110-150, Salvador, BA, Brasil. Correspondência para/Correspondence to: L. R. Sampaio. E-mail: <lramos@ufba.br>.

2 Autônoma, Brasília, DF; Curso de Especialização em Nutrição Clínica, Escola de Nutrição, Universidade Federal da Bahia. Salvador, BA, Brasil.
} 
54 | L.R. SAMPAIO \& V.C. FIGUEIREDO

no idoso ( $r=0,63 ; p<0,001)$; e para o feminino, entre os grupos: adultas $(r=0,66 ; p<0,001)$ e idosas $(r=0,34$; $p<0,001)$.

\section{Conclusão}

O índice de massa corporal apresentou correlação positiva e forte com a circunferência da cintura nos dois grupos etários e em ambos os sexos. Apesar de estatisticamente significante, o índice de massa corporal se correlacionou de forma menos intensa com a razão cintura quadril, principalmente no grupo das mulheres idosas.

Termos de indexação: adulto, antropometria, idoso, índice de massa corporal.

\section{A B S T R A C T}

\section{Objective}

This cross-sectional study investigates the correlation between the body mass index and the fat distribution anthropometric indices in adults and the elderly.

\section{Methods}

Upon ambulatory visits to the Medical School Hospital of the Federal University of Bahia (Salvador, Bahia, Brazil), 634 male and female patients (316 adults and 318 elderly subjects) were measured individually, to determine weight, height, triceps and subscapular skinfold thicknesses, and waist and hip circumferences, according to Lohman's guidelines, 1988. Pearson's correlation test was used to get the correlation, in this sample, between body mass index and fat distribution anthropometric indices.

\section{Results}

The correlations between body mass index and waist in both age groups for male were: adults $(r=0.93$; $p<0.001)$ and elderly $(r=0.89 ; p<0.001)$. For the female groups, these correlations were: adults $(r=0.93$; $p<0.001)$ and elderly $(r=0.86 ; p<0.001)$. The correlation between body mass index and waist-to-hip ratio was smaller, but statistically significant, between these groups: thus, for male adults ( $r=0.64 ; p<0.001)$ and elderly $(r=0.63 ; p<0.001)$; and for female adults $(r=0.66 ; p<0.001)$ and elderly $(r=0.34 ; p<0.001)$.

\section{Conclusion}

Body mass index was found to correlate strongly and positively with waist circumference in both age groups, for both genders. Though statistically significant, Body mass index showed a weaker correlation with waist-to-hip ratio, primarily in the elderly women's group.

Indexing terms: adult, anthropometry, aged, body mass index.

\section{N T R O D U Ç Ã O}

Com o envelhecimento ocorre aumento e redistribuição do tecido adiposo. Há redução desse tecido nos membros e progressivo acúmulo na cavidade abdominal ${ }^{1,2}$. Ainda não são bem conhecidas as conseqüências dessas alterações da composição e distribuição de gordura corporal, para o processo de escolha de indicadores antropométricos que identifiquem risco nutricional no idoso.

O índice de massa corporal (IMC) é um dos indicadores antropométricos mais utilizados na identificação de indivíduos em risco nutricional. Isso ocorre em virtude da sua facilidade de aplicação, seu baixo custo e pequena variação intra ou intermedidor ${ }^{3}$. Todavia, como indicador 
de risco no idoso, ele tem sido considerado pobre, em razão de não refletir, principalmente, a distribuição regional de gordura que ocorre com o processo de envelhecimento 1 .

Por outro lado, estudos realizados em países desenvolvidos, com amostras representativas de indivíduos adultos de ambos os sexos, demonstraram que o IMC correlaciona-se bem com indicadores antropométricos de gordura não visceral (prega cutânea subescapular e prega cutânea triciptal) e de gordura abdominal ou visceral (circunferência da cintura), além de ter relação direta com a massa de gordura corporal total ${ }^{4}$. Em idosos, os estudos são escassos, não existindo, portanto, dados suficientes afirmando que essa associação permaneça com o envelhecimento.

É nesse contexto, e sabendo-se que o IMC correlaciona-se fortemente com os indicadores de distribuição de gordura na população adulta, que o presente trabalho propõe avaliar se esta associação é mantida com o envelhecimento.

\section{CASUÍSTICA E MÉTODOS}

O presente estudo foi realizado nos ambulatórios do anexo do Hospital das Clínicas da Universidade Federal da Bahia, no período de janeiro a maio de 2002. O delineamento escolhido para o desenvolvimento deste trabalho, foi do tipo transversal. Foram estudados 634 pacientes, sendo 316 adultos (132 homens e 186 mulheres de 20 a 59 anos) e 318 idosos ( 94 homens e 222 mulheres com idade $\geq 60$ anos), cujos IMC variaram de 15,2 a $45,0 \mathrm{~kg} / \mathrm{m}^{2}$. A seleção dos pacientes foi feita de acordo com a ordem de atendimento nos ambulatórios e consentimento verbal dos mesmos, considerando-se, como critério de inclusão, não apresentar qualquer alteração que impossibilitasse a verificação das medidas antropométricas.

O estudo foi aprovado pelo Comitê de Ética do Hospital das Clínicas da Universidade Federal da Bahia.
Foram verificadas as seguintes medidas antropométricas: peso, altura, pregas cutâneas, triciptal (PCT) e subescapular (PCSE), circunferências da cintura (CC) e do quadril (CQ).

As medidas foram duplicadas, utilizando-se a média aritmética, e uma terceira medida foi verificada, quando os dois primeiros valores apresentaram diferenças não aceitáveis ${ }^{5}$.

O peso foi verificado a partir de balança digital (marca Filizola, com capacidade de $150 \mathrm{~kg}$ e intervalo de $100 \mathrm{~g}$ ) com o indivíduo descalço e usando roupas leves. A altura foi verificada utilizando-se um antropômetro vertical fixo à balança.

Foram verificadas as circunferências da cintura e quadril, com o auxílio de uma fita métrica inelástica (TBW Importadora Ltda.). A circunferência da cintura foi medida na cintura natural, ou seja, entre as costelas inferiores e as cristas ilíacas. A leitura foi feita no momento da expiração, e realizou-se no milímetro mais próximo. A circunferência do quadril foi verificada no nível da sínfise púbica com a fita circundando o quadril na parte mais saliente entre a cintura e a coxa e com o indivíduo usando roupas finas.

As pregas cutâneas foram verificadas com o auxílio do calibrador de pregas (marca Lange - TBW Importadora Ltda.). A prega cutânea triciptal foi verificada no ponto médio entre o processo acromial da escápula e o olecrano, na região posterior do braço. O indivíduo permaneceu com o braço relaxado, estendido e ligeiramente afastado do corpo. A prega cutânea subescapular foi verificada imediatamente abaixo do ângulo inferior da escápula na diagonal, formando um ângulo de $45^{\circ}$ entre a prega e a coluna vertebral.

Calculou-se o IMC considerando-se a razão peso atual $(\mathrm{kg})$ e o quadrado da estatura $\left(\mathrm{m}^{2}\right)$. Os pontos de corte propostos pela Organização Mundial da Saúde $(\mathrm{OMS})^{6}$, foram utilizados como critério de diagnóstico do estado nutricional.

O acúmulo de gordura abdominal foi estimado segundo os seguintes indicadores: razão cintura/quadril (RCQ), razão entre pregas (RPC) e 
circunferência da cintura isolada. Para identificação do tipo de distribuição de gordura segundo a RCQ, utilizou-se a classificação proposta por Lohman ${ }^{5}$, isto é, RCQ acima do recomendado, em mulheres, $R C Q \geq 0,85$; em homens, $R C Q \geq 1,00$.

A cintura isolada foi analisada a partir dos pontos de corte sugeridos pela Organização Mundial da Saúde ${ }^{6}$. Mulheres com valores de CC acima de $80 \mathrm{~cm}$ e homens com valores de CC superiores a $94 \mathrm{~cm}$ foram classificados como apresentando um acúmulo de gordura abdominal considerado como risco associado ao desenvolvimento de doenças ligadas à obesidade. A razão entre pregas foi avaliada em termos de média e desvio-padrão e utilizada para a análise de correlação com o IMC.

Fez-se a análise estatística por intermédio dos pacotes estatísticos Statistical Package for the Social Science (SPSS)7, versão 10.0, e Epi Info8, versão 6.0. As prevalências da amostra total foram apresentadas com intervalos de confiança de $95 \%$. O teste de correlação de Pearson foi utilizado para análise das variáveis contínuas. Considerou-se como estatisticamente significantes, os valores de $p$ menores que 0,05.
RES ULTA D OS

Para as características antropométricas dos grupos etários por sexo (Tabela 1) observa-se que as médias de idade para os adultos (20-59 anos) e idosos (60-99 anos) foram, respectivamente, de $43,3 \pm 10,7$ anos e 70,1 $\pm 6,7$ anos.

Na análise por faixa etária, dentro de cada grupo de sexo, não foi encontrada diferença estatisticamente significante, quando a média do IMC dos adultos foi comparada com a dos idosos.

As mulheres apresentaram IMC médio superior ao dos homens, nos dois grupos de idade. Quanto à medida da CC, foi verificado que a média aumentou com a idade nos dois grupos de sexo, encontrando-se uma diferença de $3,5 \mathrm{~cm}$ entre os adultos e idosos do sexo masculino $(p=0,002)$ e uma diferença de $4,7 \mathrm{~cm}$ entre os adultos e idosos do sexo feminino $(p=0,001)$.

A média da RCQ foi, também, estatisticamente superior nos idosos de ambos os sexos, quando comparada à média da RCQ dos adultos.

Ainda na Tabela 1, observa-se que, apesar de os valores médios correspondentes à RPC dos idosos serem mais elevados, apenas no sexo

Tabela 1. Características antropométricas segundo sexo e faixa etária (média, desvio-padrão, valores mínimo e máximo).

\begin{tabular}{|c|c|c|c|c|c|c|c|c|c|c|c|}
\hline \multirow{3}{*}{ Variáveis } & & & & \multicolumn{7}{|c|}{ Masculino } & \multirow{3}{*}{$p$ valor } \\
\hline & \multicolumn{3}{|c|}{ Total $(n=634)$} & \multicolumn{3}{|c|}{ Adulto $(n=132)$} & \multicolumn{4}{|c|}{ Idoso $(n=94)$} & \\
\hline & M & & DP & M & & DP & M & & & $\mathrm{DP}$ & \\
\hline Idade (anos) & 56,60 & \pm & 16,10 & 41,90 & $\pm 10,60$ & $(20,0-59,0)$ & 70,50 & \pm & 7,50 & $(60,0-99,0)$ & - \\
\hline $\mathrm{IMC}\left(\mathrm{kg} / \mathrm{m}^{2}\right)$ & 26,30 & \pm & 5,00 & 24,80 & 4,70 & $(17,0-39,7)$ & 24,40 & \pm & 3,20 & $(15,2-33,1)$ & 0,520 \\
\hline $\mathrm{CC}(\mathrm{cm})$ & 87,50 & \pm & 12,10 & 86,10 & $\pm 12,60$ & $(65,2-26,3)$ & 89,60 & \pm & 9,10 & $(65,5-110,0)$ & 0,020 \\
\hline $\mathrm{RCQ}$ & 0,91 & \pm & 0,09 & 0,92 & 0,08 & $(00,7-01,2)$ & 0,97 & \pm & 0,07 & $(00,8-01,1)$ & 0,000 \\
\hline \multirow[t]{4}{*}{ RPC } & 1,32 & \pm & 0,50 & 1,75 & 0,50 & $(00,8-03,3)$ & 1,87 & \pm & 0,50 & $(00,9-03,2)$ & 0,100 \\
\hline & & & & \multicolumn{7}{|c|}{ Feminino } & \\
\hline & & & & \multicolumn{3}{|c|}{ Adulto $(n=186)$} & \multicolumn{4}{|c|}{ Idoso $(n=222)$} & \\
\hline & & & & $M$ & & $\mathrm{DP}$ & $M$ & & & $\mathrm{DP}$ & \\
\hline Idade (anos) & 56,60 & \pm & 16,10 & 44,30 & $\pm \quad 10,70$ & $(20,0-59,0)$ & 69,90 & \pm & 6,30 & $(60,0-090,0)$ & - \\
\hline IMC $\left(\mathrm{kg} / \mathrm{m}^{2}\right)$ & 26,30 & \pm & 5,00 & 27,10 & 5,80 & $(14,4-44,1)$ & 27,30 & \pm & 4,80 & $(16,1-045,0)$ & 0,650 \\
\hline $\mathrm{CC}(\mathrm{cm})$ & 87,50 & \pm & 12,10 & 84,80 & $\pm \quad 13,70$ & $(58,0-125,2)$ & 89,50 & \pm & 10,80 & $(61,5-115,0)$ & 0,000 \\
\hline $\mathrm{RCQ}$ & 0,91 & \pm & 0,09 & 0,85 & 0,08 & $(00,7-01,1)$ & 0,92 & \pm & 0,08 & $(00,7-001,1)$ & 0,000 \\
\hline RPC & 1,32 & \pm & 0,50 & 1,00 & 0,20 & $(00,5-02,0)$ & 1,10 & \pm & 0,30 & $(00,5-002,7)$ & 0,001 \\
\hline
\end{tabular}

IMC= Índice de massa corporal; $C C=$ Circunferência da cintura; RCQ= Razão cintura quadril; RPC= Razão entre pregas cutâneas. 
feminino foi encontrada diferença estatisticamente significante entre os grupos etários (mulher adulta $\mathrm{RPC}=1$ e mulher idosa $\mathrm{RPC}=1,1 ; p=0,001)$.

Entre as relações do índice de massa corporal com a circunferência da cintura, segundo a faixa etária, por grupo de sexo, observa-se (Figura 1) a forte associação entre IMC e CC nos dois grupos etários do sexo masculino (adulto $r=0,93, r^{2}=0,86 ; p<0,001$ e idoso $r=0,89$, $\left.r^{2}=0,80 ; p<0,001\right)$. Para o grupo das mulheres, os resultados foram semelhantes, ou seja, a correlação entre essas variáveis também foi elevada nos dois grupos etários (adulta $r=0,93$, $r^{2}=0,86 ; p<0,001$ e idosa $r=0,86, r^{2}=0,73$; $p<0,001)$.

Quanto à correlação entre o IMC e a razão cintura quadril, os resultados obtidos evidenciam uma associação também estatisticamente significante, mas não tão forte quanto a encontrada com a CC, nos dois grupos etários de ambos os sexos (Figura 2). Vale ressaltar que, para um dado valor da RCQ, os adultos do sexo masculino apresentam valores diferentes de IMC, demonstrando grande variação inter-indivíduos neste grupo.

Nota-se ainda que, no grupo das mulheres, as idosas apresentaram o menor valor de $r(0,34)$ para esta relação, sendo o poder explicativo da associação de apenas $11 \%$.

Verifica-se, portanto, que o IMC não se correlacionou tão bem com a RCQ nos dois grupos etários e em ambos os sexos, quanto se correlacionou com a CC, e que a menor correlação foi encontrada no grupo das mulheres idosas.

Por outro lado, a correlação entre IMC e a razão entre pregas foi negativa nos dois grupos etários do sexo masculino. Apesar de no adulto esta relação ter sido estatisticamente significante, o poder explicativo da relação foi de apenas 7\%, evidenciando uma fraca correlação (Figura 3).

No grupo dos homens idosos, a correlação não foi estatisticamente significante $(r=-0,08$, $\left.r^{2}=0,007 ; p=0,43\right)$. Para o sexo feminino, não houve significância estatística na relação entre o IMC e a RPC, tanto no grupo das adultas, quanto
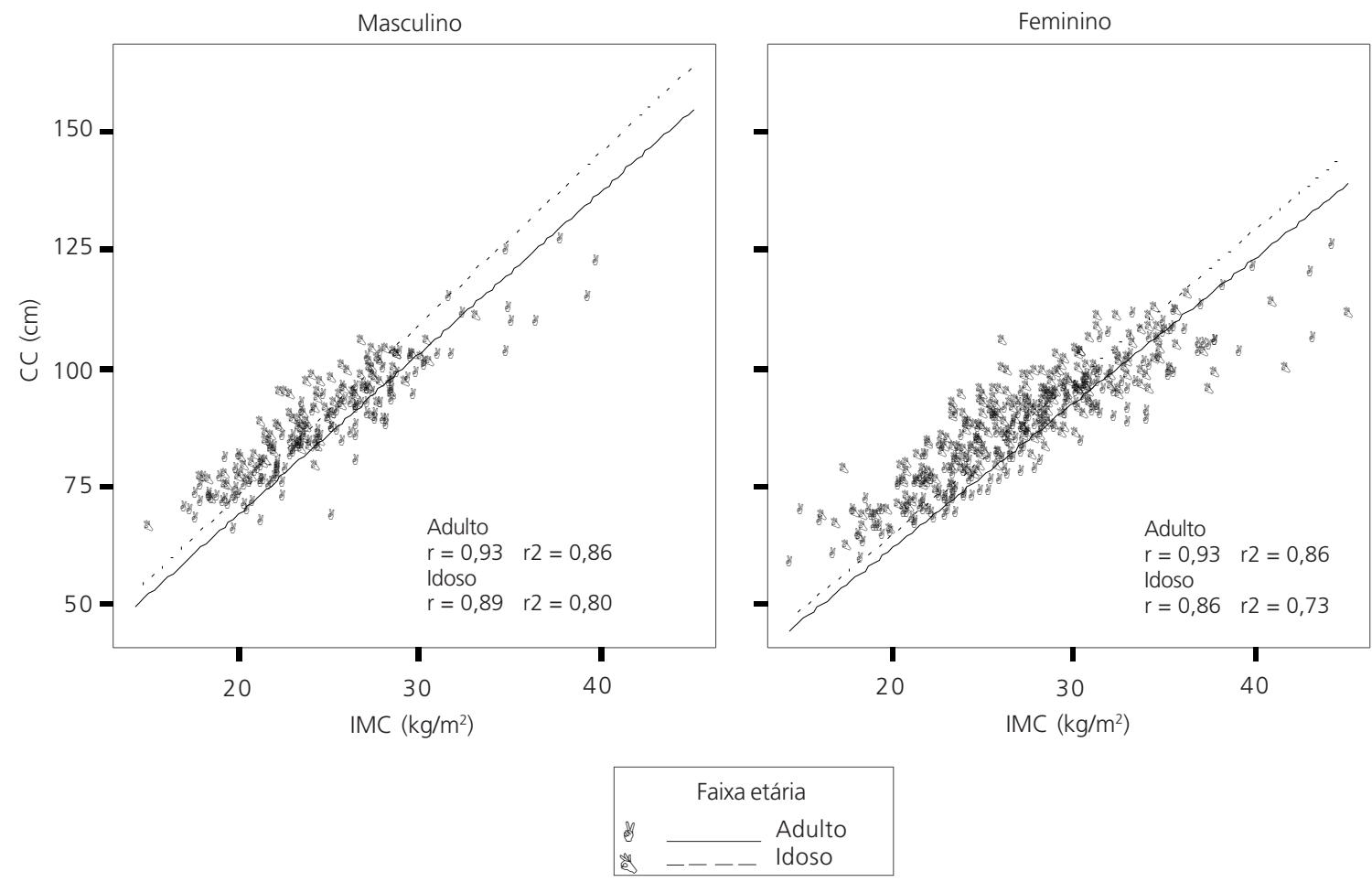

Figura 1. Correlação entre o índice de massa corporal (IMC) e circunferência da cintura (CC) em ambos os sexos, segundo faixa etária. 
no das idosas, o que significa uma correlação extremamente fraca entre essas variáveis, nas duas faixas etárias (Figura 3).

No sentido de compreender as diferenças nas correlações entre o IMC e os dois principais indicadores de acúmulo de gordura abdominal (CC e RCQ), calculou-se o percentual de indivíduos apresentando valores de CC e RCQ superiores ao recomendado, segundo o estado nutricional determinado pelo IMC (Tabela 2). Observa-se que, quase metade das mulheres idosas eutróficas, apresentaram acúmulo de gordura abdominal segundo a CC, contra 13,6\% das mulheres adultas.

Os homens eutróficos, dos dois grupos etários, praticamente não apresentaram acúmulo de gordura abdominal, segundo esse indicador.

Por outro lado, as mulheres adultas (12,5\%) e as idosas (25,0\%), com diagnóstico de magreza,
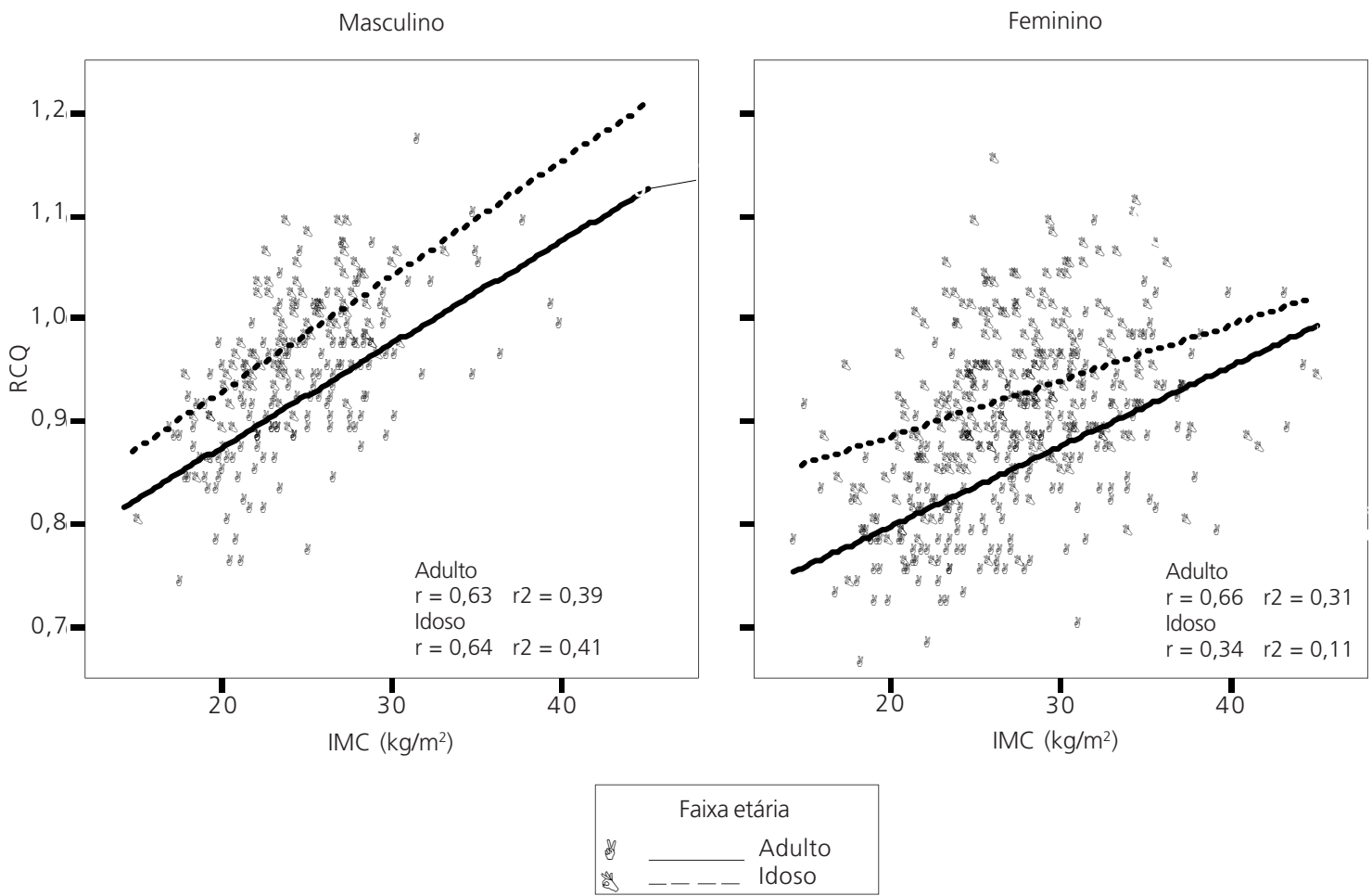

Figura 2. Correlação entre o índice de massa corporal (IMC) e a razão cintura quadril (RCQ) em ambos os sexos, segundo faixa etária.

Tabela 2. Percentagens de indivíduos com valores da CC e RCQ superiores ao recomendado segundo estado nutricional, faixa etária e sexo.

\begin{tabular}{|c|c|c|c|c|c|c|c|c|c|c|c|c|c|c|c|c|}
\hline & \multicolumn{8}{|c|}{ Masculino } & \multicolumn{8}{|c|}{ Feminino } \\
\hline & \multicolumn{4}{|c|}{ Adulto } & \multicolumn{4}{|c|}{ Idoso } & \multicolumn{4}{|c|}{ Adulto } & \multicolumn{4}{|c|}{ Idoso } \\
\hline & \multicolumn{2}{|c|}{$C C$} & \multicolumn{2}{|c|}{$\mathrm{RCQ}$} & \multicolumn{2}{|c|}{ CC } & \multicolumn{2}{|c|}{$\mathrm{RCQ}$} & \multicolumn{2}{|c|}{ CC } & \multicolumn{2}{|c|}{$\mathrm{RCQ}$} & \multicolumn{2}{|c|}{$C C$} & \multicolumn{2}{|c|}{$\mathrm{RCQ}$} \\
\hline & $\mathrm{n}$ & $\%$ & $n$ & $\%$ & $n$ & $\%$ & $n$ & $\%$ & $\mathrm{n}$ & $\%$ & $\mathrm{n}$ & $\%$ & $\mathrm{n}$ & $\%$ & $\mathrm{n}$ & $\%$ \\
\hline Magreza & - & - & - & - & - & - & - & - & - & - & 1 & 12,5 & - & - & 2 & 25,0 \\
\hline Eutrofia & - & - & 4 & 6,6 & 3 & 5,8 & 11 & 21,2 & 9 & 13,6 & 15 & 22,7 & 33 & 49,3 & 47 & 70,1 \\
\hline Sobrepeso & 18 & 37,5 & 8 & 16,7 & 25 & 71,4 & 17 & 48,6 & 40 & 78,4 & 28 & 54,9 & 83 & 98,8 & 79 & 94,0 \\
\hline Obesidade & 14 & 100,0 & 8 & 57,1 & 4 & 100,0 & 3 & 75,0 & 61 & 100,0 & 47 & 77,0 & 63 & 100,0 & 59 & 93,7 \\
\hline
\end{tabular}

$C C=$ circunferência da cintura; $R C Q$ = razão cintura quadril. 

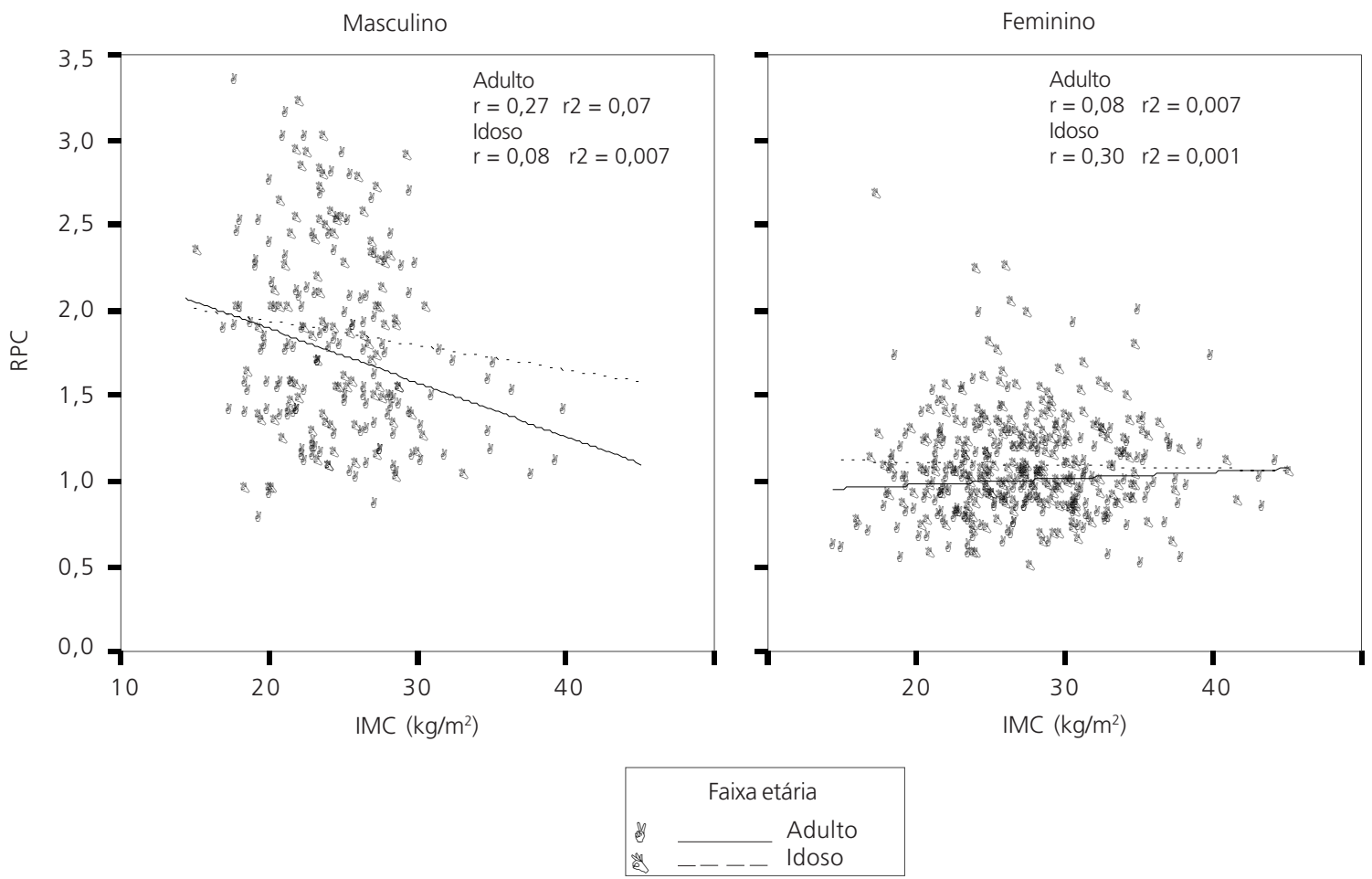

Figura 3. Correlação entre o Indice de massa corporal (IMC) e a razão entre pregas (RPC) em ambos os sexos, segundo faixa etária.

já apresentavam acúmulo de gordura abdominal segundo a RCQ. Quanto aos indivíduos eutróficos, $70,1 \%$ das mulheres idosas já apresentavam acúmulo de gordura abdominal, contra $22,7 \%$ das mulheres adultas. Os percentuais encontrados no sexo masculino foram inferiores nos dois grupos etários (adultos 6,6\%; idosos 21,2\%), mas superiores aos encontrados para a CC (Tabela 2).

Isso significa que a correlação menos forte entre o IMC e a RCQ, principalmente no grupo dos idosos, pode ser explicada pela identificação de excesso de gordura abdominal segundo RCQ, em indivíduos magros ou eutróficos.

\section{I S C U S S Ã O}

No presente estudo transversal, foi investigada a correlação entre o IMC e os indicadores de distribuição de gordura entre adultos e idosos. Os resultados foram analisados dentro de cada grupo de sexo e comparados com estudos, na sua maioria, realizados em adultos, ou envolvendo análise conjunta de adultos e idosos, em razão da escassez de estudos específicos com idosos.

Os resultados do presente estudo evidenciaram forte correlação entre o IMC e a CC, nos dois grupos etários e em ambos os sexos. Esses achados coincidem com resultados da literatura. Zamboni et al. ${ }^{9}$, avaliando indivíduos de 27 a 78 anos, observaram que, nos homens, a correlação do IMC com a CC foi de $r=0,92$ $(p<0,001)$ e nas mulheres foi de $r=0,75(p<0,001)$.

Em estudos realizados apenas com indivíduos adultos, foram encontradas correlações entre o IMC e a CC, que variaram de $r=0,76$ a $r=0,94$, mostrando excelente associação entre essas variáveis, nesse grupo etário ${ }^{10-12}$.

Em um dos poucos estudos com amostras constituídas apenas por indivíduos idosos, Goodman-Gruen \& Barrett-Connor ${ }^{13}$ verificaram as diferenças por sexo nas medidas de gordura 
corporal e de distribuição de gordura corporal, encontrando, também, forte correlação entre o IMC e a CC, em ambos os sexos (homens: $r=$ 0,$86 ; p=0,0001$ / mulheres: $r=0,81 ; p=0,0001$ ). Portanto, independentemente do grupo etário e sexo, o IMC apresenta uma boa correlação com a CC.

Quanto à relação entre o $\mathrm{IMC}$ e a $\mathrm{RCQ}$, observou-se, no presente estudo, que a correlação nos dois grupos etários e, em ambos os sexos, foi estatisticamente significante; entretanto, não foi tão forte como a correlação identificada com a circunferência da cintura. Outros estudos apontam resultados semelhantes: Shimokata et al. ${ }^{14}$, avaliando indivíduos de 17 a 96 anos de idade, detectaram uma correlação de $r=0,56$ entre $o$ IMC e a RCQ em homens, e de $r=0,41 \mathrm{em}$ mulheres. Em estudo com adultos e idosos, Seidell et al. ${ }^{15}$ identificaram uma correlação de $r=0,68$ $(p<0,001)$ em homens, de $r=0,47(p<0,05)$, em mulheres. Zamboni et al. ${ }^{9}$ e Armellini et al. ${ }^{10}$ encontraram correlações de $r=0,17(p<0,05)$ e $r=0,37$, respectivamente, em mulheres adultas.

Goodman-Gruen \& Barrett-Connor ${ }^{13}$ identificaram correlação mais fraca do que a encontrada no nosso estudo, entre o IMC e a RCQ $(r=0,22 ; p=0,001)$ nas mulheres idosas. Ao contrário do encontrado em nosso estudo, esses autores não observaram, nos homens idosos, relação estatisticamente significante $(r=0,13$; $p=0,14)$ entre essas variáveis.

Nosso estudo mostrou que, apesar de as correlações entre o IMC e a CC, e do IMC com a $R C Q$, nos dois grupos etários, serem estatisticamente significantes, nas idosas essas correlações foram menos fortes. Provavelmente, a razão esteja no fato de que quase metade das idosas eutróficas foram diagnosticadas como apresentando acúmulo de gordura abdominal, segundo a CC, e idosas magras ou eutróficas foram diagnosticadas como apresentando acúmulo, segundo a RCQ.

Os estudos que analisam a relação entre 0 IMC e as pregas isoladas apontam forte correlação entre essas variáveis. Entretanto, quando a análise é feita com a razão entre as pregas, a correlação passa a ser extremamente fraca. Segundo Goran et al. ${ }^{16}$, isto ocorre, provavelmente, devido à pequena variação nos valores da razão. Esta explicação talvez justifique os resultados encontrados em nosso estudo, quando da análise dessas variáveis.

Conclui-se, portanto, que: tanto no grupo dos adultos quanto no dos idosos, de ambos os sexos, a correlação entre o IMC e a CC foi elevada. As mulheres idosas apresentaram menores correlações entre o IMC e os indicadores de gordura corporal e a diferença mais relevante nas correlações entre os grupos etários, ocorreu entre o IMC e a RCQ, no grupo do sexo feminino. O IMC se correlacionou de forma menos forte com a RCQ, do que com a CC, nos dois grupos etários e em ambos os sexos.

\section{REFERÊ NCIAS}

1. Perissinotto E, Pisent C, Sergi G, Grigoletto F, Enzi $G$. Anthropometric measurements in the elderly: age and gender differences. Br J Nutr. 2002; 87(2):177-86.

2. Seidell JC, Visscher TLS. Body weight and weight change and their health implications for the elderly. Eur J Clin Nutr. 2000; 54(Suppl 3):533-9.

3. Anjos LA. Indice de massa corporal (massa corporal estatura ${ }^{2}$ ) como indicador de estado nutricional de adultos: revisão de literatura. Rev Saude Publica. 1992; 26(6):431-6.

4. Navarro AM, Marchini JS. Uso de medidas antropométricas para estimar gordura corporal em adultos. Nutrire: Rev Soc Bras Alimen Nutr. 2000; 19/20:31-47.

5. Lohman TG, Roche AE, Martorell R. Anthropometric standardization reference manual. Illinois: Human Kinetics Books; 1988.

6. World Health Organization. Obesity: Preventing and managing the global epidemia. Geneve; 1997.

7. Statistical Package for the Social Sciences - version 10.0, 1999. 
8. Dean JÁ, Dean AG, Burton AH, Dicker RC. Epillnfo [computetr version]. Version 6.0: a word processing database and statistics program for epidemiology on microcomputers. Atlanta, Georgia: Centers of Disease Control and Prevebtions; 1995.

9. Zomboni M, Turcanato E, Armellini F, Zivelonghi A, Santana $H$, Bergano-Andreis IA, et al. Sagittal abdominal diameter as a practical predictor of visceral fat. Int J Obes Relat Metab Disord. 1998; 22(7):655-60.

10. Armellini F, Zamboni M, Castelli S, Micciolo R, Minor A, Turcanato E, et al. Measured and predicted total and visceral adipose tissue in women. Correlations with metabolic parameters. Int J Obes Relat Metab Disord. 1994; 18(9):641-7.

11. Pouliot M-C, Després J-P, Lemieux S, Moorjani $S$, Bouchard C, Tremblay A, et al. Waist circunference and abdominal sagittal diameter: best simple anthropometric indexes of abdominal visceral adipose tissue accumulation and related cardiovascular risk in men and women. Am J Cardiol. 1994; 73(1):460-8.

12. Richelsen B, Pederson SB. Associations between different anthropometric measurements of fatness and metabolic risk parameters in non-obese, healthy, middle-aged men. Int J Obes Relat Metab Disord. 1995; 19(3):169-74.

13. Goodman-Gruen D, Barret-Connor E. Sex differences in measures of body fat and body fat distribution in the elderly. Am J Epidemiol. 1996; 143 (9):898-906.

14. Shimokata H, Tobin JD, Muller DC, Elahi D, Coon PJ, Andres R. Studies in the distribution of body fat: I. Effects of age, sex and obesity. J Gerontology. 1989; 44(2):M65- M73.

15. Seidell JC, Cigolini M, Charzewsla J, Ellinger BM, Deslypere J, Cruz A. Fat distribution in European men: a comparison from anthropometric measurements in relation to cardiovascular risk factors. Int J Obes Relat Metab Disord.1992; 16(1):17-22.

16. Goran MI, Allison DB, Poehlman ET. Issues relating to normalization of body fat content in men and Women. International. Int J Obes Relat Metab Disord. 1995; 19(9): 638-43.

Recebido para publicação em 25 de agosto de 2003 e aceito em 14 de abril de 2004 